\title{
Curaciones de Heridas Crónicas: Reto para el Cuidado en la Enfermería
}

\section{Treatment for Chronic Wounds: Have Become a Challenge for Nursing}

\author{
Janeth Jinete Acendra ${ }^{1}$ \\ Universidad Metropolitana \\ Rosa Morales Aguilar ${ }^{2}$ \\ Universidad Metropolitana \\ Gloria Lastre Amell ${ }^{3}$ \\ Universidad Simón Bolívar
}

\begin{abstract}
RESUMEN
Introducción: Las curaciones de las heridas crónicas se han convertido en un reto para la Enfermería. Las nuevas tendencias y avances tecnológicos en el manejo de estas heridas demandan la atención respecto al cuidado que se les debe brindar a los pacientes para mejorar su calidad de vida. Las heridas crónicas más prevalentes son las úlceras por presión y las de pie diabético. Objetivo: Identificar las curaciones de heridas crónicas realizadas por el personal de Enfermería en servicios de medicina interna de un hospital de la ciudad de Barranquilla. Materiales y métodos: Estudio descriptivo transversal en 51 enfermeras de los servicios de medicina interna de un hospital de Barranquilla. Como instrumento de medición, se usó un test de curación de heridas y los datos obtenidos se analizaron mediante el paquete estadístico SPSS 20. Resultados: El 100\% de las enfermeras utilizan el tipo de curación convencionaly el tipo de heridas crónicas más común es el pie diabético, con un $73 \%$. Conclusión: Se identificó que la herida crónicapredominante en los servicios de medicina interna de un hospital de Barranquilla fue el pie diabético, y el tipo de curaciones que realizó el personal de enfermería fue la curación convencional, debido a que en esta instituciónno cuentan con recursos necesarios para realizar las curaciones con alta tecnología. Aun así, el personal no desconoce que la curación con tecnología es eficaz.
\end{abstract}

Palabras Clave: enfermería, heridas, curación, tecnología (Fuente: DeCS).

\section{ABSTRACT}

Introduction: Treatments for chronic wounds have become a challenge for nursing. New trends and technological advances in the treatment of these wounds demand attention regarding the care that must be afforded to patients to improve their quality of life. The most prevalent chronic wounds are pressure wounds and those related to gangrene. Objective: To identify the treatments for chronic wounds achieved by nursing personnel in the internal medicine services of a hospital in the city of Barranquilla. Materials and methods: A descriptive cross-sectional study of 51 nurses in the internal medicine services of a hospital in the city of Barranquilla. A test of injury treatments was used as an instrument of measurement and the data received was analyzed through the SPSS 20 statistical package. Results: $100 \%$ of the nurses used traditional treatments and the most common chronic injury was diabetic foot, at $73 \%$. Conclusion: It was identified that the predominant chronic injury in the in the internal medicine services of the hospital in Barranquilla was diabetic foot, and the type of treatments that the nursing staff used was traditional, due to the fact that in that institution they do not access to the necessary resources to carry out the treatments with high technology. Nevertheless, the staff is aware that treatment with technology is efficient.

Keywords: nursing, wounds, treatment, technology (Source: MeSH, NLM).

Enfermera. Especialista en Modelos, Tipos y Diseños de Investigación. Terapista enterostomal.

Enfermera. Maestrante en Enfermería. Especialista en Cardiología.

Enfermera. MSc en Salud Pública. Especialista en Auditoria en Salud. Correo electrónico: glastre@unisimonbolivar.edu.co 


\section{Introducción}

A través de la historia, las heridas han sido un tema muy trascendental que han acompañado año hombre desde su inicio en el recorrido desde aproximadamente 5000 años A.C. Es por ello que los egipcios, aplicaban curaciones compuestas de grasa animal, miel y fibras de algodón. Sin saberlo, estaba aplicando una curación no adherente, antibacteriana, osmótico enzimática y finalmente absorbente al exudado, estas curaciones se han aplicado desde mucho tiempo (1).

Entre las heridas, merecen particular atención las Heridas Crónicas (HC), que se caracterizan por la nula o escasa tendencia a la cicatrización espontánea, y requieren un período de cicatrización mayor que de las heridas agudas. Esta característica las constituyeen un problema de salud importante, que afecta generalmente a las personas mayores, y en especial a las que presentan situaciones que afectan su movilidad. La población anciana presenta una mayor susceptibilidad a padecer algunos tipos de HC, como las úlceras por presión o las úlceras vasculares (2).

Las HC más prevalentes son las úlceras por presión y el pie diabético. Las primerasson aquellas lesiones cutáneas que se producen a consecuencia de una falta de irrigación sanguínea y de una irritación de la piel que recubre una prominencia ósea. Generalmente, aparecen en zonas que hansufrido la presión de una cama, silla de ruedas, molde, férula u otro objeto rígido durante un periodo prolongado de tiempo, independientemente de la posición en la que permanezca la persona (3).

Las personas que sufren úlceras por presión en particular, y HC en general, constituyen hoy en día un importante problema por sus repercusiones en diferentes ámbitos, tales como la afectación en la salud de quienes las padecen, el deterioro de la calidad de vida de éstos, así como la de sus cuidadores responsables, sean familiares o no. De la misma forma, inciden en el consumo de recursos que debe aportar el sistema de salud, pudiendo incluso alcanzar responsabilidades legales (4), al ser consideradas en muchas situaciones como un problema evitable.

Los recientes avances en métodos de curación de $\mathrm{HC}$, ya sea convencionales o con el uso de tecnología, han encaminado el avance de diversas particularidades terapéuticas que ofrecen esperanza a los pacientes afectados. Sin embargo, para lograr estos cambios, se requiere de metodología de trabajo específica en un rangode tiempo determinado, que permita el diseño de estrategias a implementar, así como su desarrollo y ejecución para el cumplimiento de los objetivos diseñados. Es claro, en todo caso, que la curación con tecnología resulta más eficaz y eficiente que la curación convencional.

Debido al uso de apósitos de alta tecnología, la curación con tecnología favorece la cicatrización al estimular el microambiente de la herida. La curación convencional,en cambio,usa materiales de baja absorción y alta capacidad de desecación, representados por gasa y algodón, en forma de compresas, apósitos o torundas(5). Asimismo, los avances en los estudios reconocen que las curaciones de heridas crónicas se dan sobre todo por los métodos más conocidos, que son los convencionales, en tanto que los de aplicación de tecnología son menos usados por los costos que producen al sistema de salud. Esta situaciónha llevado cada día a la búsqueda de diversas modalidades terapéuticas que alienten la esperanza a familiares y pacientes con heridas crónicas.

El método convencional se realiza en ambiente seco, utiliza apósitos pasivos, así como tópicos (antisépticos, antimicrobianos, otros) y es de frecuencia diaria o mayor. Por su parte, la curación avanzada se realiza en un ambiente húmedo fisiológico, utiliza apósitos activos, no usa tópicos en lo posible y su frecuencia depende de las condiciones locales de la herida (6).

Durante el seguimiento, se puede cambiar de apósitos, agregar o quitar antibióticos y/o espaciar, o intensificar la frecuencia de las curaciones, la práctica permite tener una mayor experiencia en este punto (7). El estudio de Andrades (7) evidencia, en este sentido, que la aplicación de apósitos mantiene un ambiente húmedo fisiológico, que se convierte en una barrera indispensable para aislar y proteger la herida, de tal manera que el paciente se sienta más cómodo desde su bienestar psicosocial.

La cicatrización de una herida o úlcera compleja no solo comprende aspectos fisiológicos y biológicos. También involucra elementos psicosociales y económicos, a los que se añade la complejidad de las heridas. Investigadores de diferentes lugares de Europa se congreraron para perfilar un documento titulado "Posicionamiento", en donde reflejaron los aportes más actualizados sobre el tema (8). 
El grupo de heridas reconocidas comopie diabéticoson las más complejas para la Enfermería, sobre todo por la alteración en niveles proteínicos de los pacientes, que son referentesclaves en el esquema de cicatrización.

Con base en lo anteriormente expuesto, este artículo tiene como objetivo identificar el tipo de curación de heridas crónicas realizadas por el personal de enfermería en el servicio de medicina interna de un hospital de Barranquilla.

\section{Materiales y Métodos}

Nuestro estudio fue de carácter cuantitativo, descriptivo-transversal. El universo estuvo conformado por 51 enfermeras entre jefes (12) y auxiliares (39) de los distintos servicios de medicina interna de un hospital de Barranquilla. Se escogió una muestra de 15 enfermeras, 9 profesionales y 6 auxiliares. El muestreo fue no probabilístico, de conveniencia. Los criterios de inclusión consistieron en que las enfermeras estuvieran certificadas con su título profesional o técnico y que laboraran en el servicio.

Se diseñó un cuestionario integrado por 11 preguntas cerradas de selección múltiple, el cual indagó sobre las características socio-demográficas, sexo, nivel de escolaridad, tipo de heridas y lacuración convencional de las HC. El análisis de la información se hizo mediante el paquete estadístico SPSS 18.

\section{Resultados}

En la Figura 1, se observa que el $100 \%$ de los sujetos de estudio son del sexo femenino.

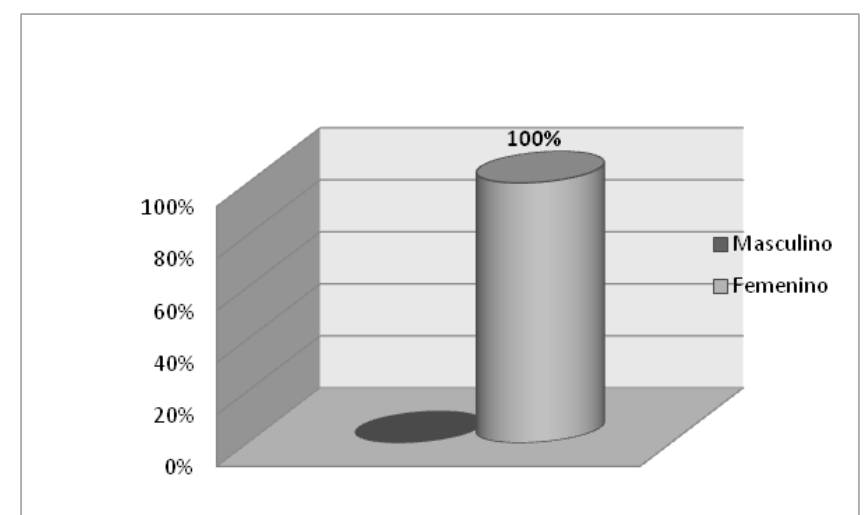

Figura 1. Características socio-demográficas del personal de enfermería (profesional y auxiliar)

Por otra parte, como muestra la Figura 2, el 60\% de los profesionales de Enfermería posee un nivel académico profesional.

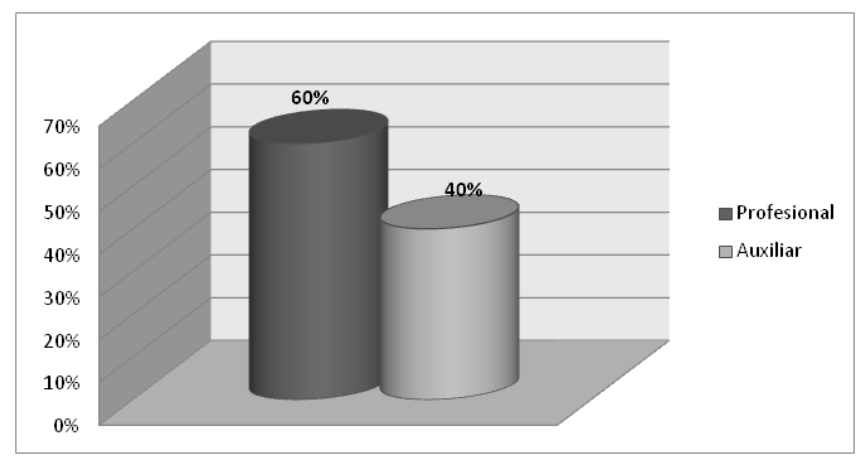

Figura 2. Nivel académico del personal de enfermería

Además, el 73\% de las enfermeras señaló que el pie diabético es la herida crónica que atiendecon mayor frecuencia en el servicio de medicina interna, seguido de las ulceras por presión (27\%).

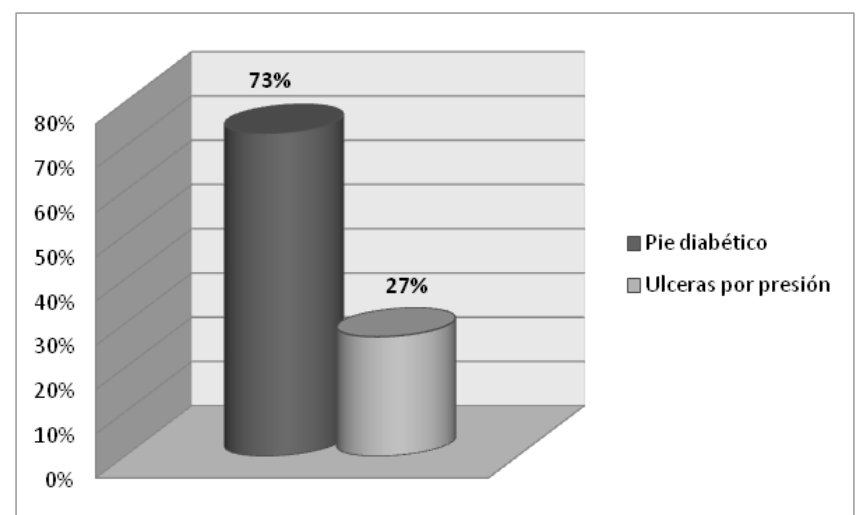


Figura 3. Tipos de heridas frecuentes en pacientesde servicio de medicina interna

El $100 \%$ de las enfermeras señaló que el tipo de curación que realizan para manejar las $\mathrm{HC}$ es la curación convencional, pero también reconocen cambios sustanciales entre el hecho de realizar una curación convencional o con tecnología.

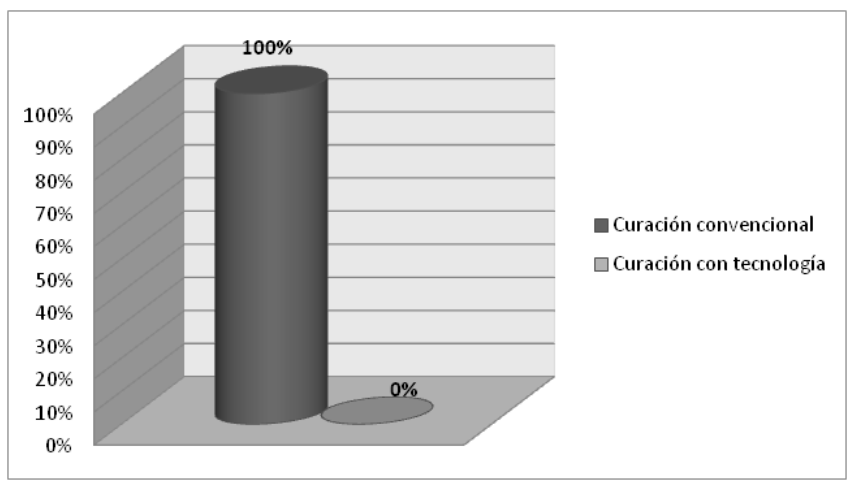

Figura 4. Tipo de curación de los pacientes según método convencional y con tecnología

\section{Discusión}

El presente estudio muestra primero que el total de las participantes pertenecen al sexo femenino. Pero el cuidado inteligente, como lo llamó Florence Nightingale, la fundadora de enfermería profesional, ese sí es solo privativo del hombre, aunque desde los tiempos más remotos de la Antigüedad ha sido una actividad fundamentalmente femenina, con excepciones durante las cruzadas de la Edad Media, cuando algunas órdenes religiosas masculinas combinaron las armas con los cuidados a los heridos y enfermos (9). Lo arrojado en este estudioevidencia que el género femenino sigue predominando en la profesión de la Enfermería.

Los resultados también indican que el nivel académico del $60 \%$ de las participantes esel profesional. Lo anterior se relaciona con la ética del cuidado, disciplina que se ocupa de las acciones responsables y de las relaciones morales entre las personas, motivadas por una solicitud, y que tiene como fin último lograr el cuidado de sus semejantes o el suyo propio (10).

Las participantes también expresaron que el tipo de curación que suelen utilizar es el convencional. Este dato se relaciona con el artículo sobre manejo avanzando de heridas (11), en el cual se asegura que hace poco tiempo el cuidado de las heridas se centraba especialmente en un tratamiento de protección de las mismas con diversos materiales como antisépticos, gasas, apósitos absorbentes, entre otros, los cuales a través del tiempo se han modificado, por lo que existen en la actualidad métodos de curación adecuados para todo tipo de lesiones. Así, a pesar de que en el siglo XXI existen curaciones avanzadas o con tecnología de punta, en nuestro medio aún predomina lo convencional. Desde esta óptica, las instituciones pertinentes deben saber que el manejo de las curaciones en heridas crónicas con elementos de tecnología de punta coadyuva a los proceso de cicatrización y calidad en atención de los usuarios.

De otro lado, las úlceras por presión deben ser consideradas el objetivo prioritario en el plan de cuidados de enfermería y, de esta manera, ella podrá convertirse en herramienta fundamental para disminuir la incidencia de eventos. Las acciones,en este sentido, deben comprender identificación del riesgo, cambios posturales, higiene e hidratación de la piel, el adecuado aporte nutricional, la protección de fuerte presión y monitorización de indicadores epidemiológicos como la incidencia y prevalencia (12).

También vale la pena resaltar lo inscrito en el estudio de Souza, el cual enuncia que la cicatrización no sólo depende de terapias tópicas, sino que en ella incidenmúltiples factores sistémicos, aunque la mayoría de las técnicas se enfoque en el manejo local de la lesión. La evolución satisfactoria de las heridas, se manifiesta contento de los profesionales de enfermería, por lo contrario si en los pacientes no se muestra evolución pueden presentar sentimientos negativos y desanimo, conduciéndolos fácilmente a estados depresivos, ideas de auto desprecio y pérdida del sentido de la vida (13). Infiriendo esto con los descritos en el articulo cuerpo heridas, vidas alteradas representaciones sociales de mujer y hombre.

Otro dato muy significativo al respecto es el reportado por Bautista, la respuesta a la variable del manejo que se proporcionaba a las úlceras por presión una vez ya presentadasindicó que el $52.4 \%$ utilizaba más de una sustancia para realizar la curación; el $26.2 \%$ usó solución salina; $14.3 \%$ empleó idodopovina, $4.8 \%$ hizo uso de agua oxigenada, y solo $2.4 \%$ aplicó agua bidestilada y otros (14). En estas 
prácticas se evidenciaque, aun siendo un servicio de cuidados intensivos, el método convencional es el más usado. Al contrastar estosdatos con nuestro estudio, se encuentra una gran similitud en cuanto a la predominancia del método de curación convencional en la enfermería.

Ramos expresa cómo en una molécula mayor de la matriz extra celular, el hiluronato tiene efectos regulatorios en la inflamación, angionesis, granulación y reepitilización en lesiones del pie diabético, por lo que representa una solida alternativa en la reducción de la amputación de miembros inferiores en la población diabética (15). Teniendo en cuenta lo anterior, se puede afirmar que los productos de tecnología aportan condiciones óptimas a heridas crónicas.Más en concreto, se dice que las técnicas avanzadas en curaciones de heridas son frutos del desarrollo tecnológico y se caracterizan por no utilizar apósitos. Entre éstas destaca la terapia con oxígeno hiperbárico y la curación de heridas con presión negativa. Y esta última se reporta como cicatrización asistida (16).

Asimismo, en este trabajo $73 \%$ de enfermeras señaló que el pie diabético es la herida crónica que atienden con mayor frecuencia en servicio de medicina interna, seguido úlceras por presión (27\%) (16). Estos datos comprueban que el píe diabético sigue siendo prevalente en pacientes con heridas crónicas por los factores asociados de su patología y se relacionan con lo hallado en el estudio de Markuson (17), en cuya población, el $47.6 \%$ debía tales úlceras a algún aspecto neuropático o relacionado con cardiopatías isquémicas; el $17,5 \%$, a una insuficiencia arterial venosa; el $17.5 \%$, a un aspecto neuropático mixto por presión venosa o arterial; el $12.7 \%$ se relacionó con la presión y el $4.7 \%$, con otros.

Se concluye, finalmente, que el cuidado oportuno de enfermería es determinante para establecer planes de cuidado encaminados a brindar atención de calidad a los pacientes. Para ello, es importante el conocimiento científico, fundamentado en conceptos teóricos y tecnológicos, en el cuidado. Tal es el caso de la curación de heridas crónicas, la cual, más allá que una conducta de rutina debe asumirse como un avance del conocimiento en el quehacer profesional. El propósito de la Enfermería se resume en promover la vida, prevenir la enfermedad, intervenir en el tratamiento y la rehabilitación, y brindar apoyo emocional tanto al paciente como al grupo familiar. Por lo tanto, el profesional de este campo debe fundamentar su práctica en conocimientos sólidos y actualizados y es necesario revisar los procesos de curación en la institución en estudio. Todo ello con el fin de actualizar a los profesionales de Enfermería para que apliquen métodos tecnológicos en las curaciones, sin dejar necesariamente de lado los métodos convencionales.

\section{Referencias}

1. Ramírez AR, Dagnino UB. Curación de heridas. Antiguos conceptos para aplicar y entender su manejo avanzado. Cuad.cir. 2006; 20 (1): 92-99.

2. Ferrer SM, Chirveches PE, Molist SG, Molas PM, Besoli CA, Jaumira AE, et al. Prevalencia de las heridas crónicas en una comarca de la provincia de Barcelona. Enferm Clin. 2009; 19:4-10.

3. Freer BE. Las ulceras de presión. Acta méd. costarric. 2001; 43(2): 47-47.

4. Gago FM, García GR, López CP, Verdu SJ, Blanco CE, Chorat S. Evaluación de la tecnología sanitaria. Apósitos basados en la cura en ambiente húmedo para heridas crónicas. Gerokomos. 2008;19 (4): 200-206.

5. Jiménez CE. Curación avanzada de heridas. Rev. Colomb. cir. 2008; 23(3): 146-155.

6. Carreño CJ. Curación avanzada de heridas.Rev. Medicina. 2008; 14 (3): 262-267.

7. Andrades P, Sepúlveda S, Gonzales J. Curación avanzada. Rev. Chilena de Cirugía. 2004; 56 (4): 396-403.

8. Fernández MJ. Heridas de difícil cicatrización. Instituto de angiología. 2011.

9. Amaro CM. Historia de la enfermería. La Habana: Editorial Ciencias Médicas; 2004.

10. Alvarado GA. La ética del cuidado. Aquichan. 2004; 4 (4): 30-39.

11. Flores MI. Manejo avanzado de heridas. Rev Mex Enf Cardiol. 2006; 14 (1): 24-28.

12. Valero CH, Parra D, Gómez RR, Camargo FF. Prevalencia de ulceras por presión en una institución de salud. Salud UIS. 2011; 43 (3): 249255. 
13. Souza CE, Santos PM, Casado AE. Cuerpos heridos, vida alterada: representaciones sociales de mujeres y hombres. Index Enferm. 2011; 20(1-2): 31-35.

14. Bautista PL, Esparza GM, Ortega AJ. Las úlceras por presión en pacientes sometidos a ventilación mecánica en la unidad de cuidados intensivos e intermedios del Iner. Rev. Inst. Nal. Enf. Resp. Mex. 2004; 17(2): 91-99.

15. Ramos CF, Velázquez MA, Castañeda AI. Efecto del hialuronato de zinc sobre las úlceras en pacientes con diabetes. Gerokomos 2007; 18(2): $38-48$.

16. VojvodicHI, Zevallos BO. Curación de heridas con presión negativa: fundamentos e indicaciones. Rev. Per. Obst Enf. 2010; 6 (1): 46-57.

17. Markuson M, Hanson D, Anderson J, Langemo D, Hunter S, Thompson $\mathrm{P}$, et al. The relationship between hemoglobin A1cvaluesand healing time for lower extremity ulcersin individuals with diabetes. Adv Skin Wound Care. 2009; 22(8): 365-372. 